\title{
Few-body quantum method in a $d$-dimensional space
}

\author{
E. Garrido ${ }^{\mathrm{a}}$, A.S. Jensen ${ }^{\mathrm{b}}$, R. Álvarez-Rodríguez ${ }^{\mathrm{c}}$ \\ ${ }^{a}$ Instituto de Estructura de la Materia, IEM-CSIC, Serrano 123, E-28006 Madrid, Spain \\ ${ }^{b}$ Department of Physics and Astronomy, Aarhus University, DK-8000 Aarhus C, Denmark \\ ${ }^{c}$ Escuela Técnica Superior de Arquitectura, Universidad Politécnica de Madrid, Avda. Juan Herrera 4, 28040 Madrid, Spain
}

\begin{abstract}
In this work we investigate the continuous confinement of quantum systems from three to two dimensions. Two different methods will be used and related. In the first one the confinement is achieved by putting the system under the effect of an external field. This method is conceptually simple, although, due to the presence of the external field, its numerical implementation can become rather cumbersome, especially when the system is highly confined. In the second method the external field is not used, and it simply considers the spatial dimension $d$ as a parameter that changes continuously between the ordinary integer values. In this way the numerical effort is absorbed in a modified strength of the centrifugal barrier. Then the technique required to obtain the wave function of the confined system is precisely the same as needed in ordinary three dimensional calculations without any confinement potential. The case of a two-body system squeezed from three to two dimensions is considered, and used to provide a translation between all the quantities in the two methods. Finally we point out perspectives for applications on more particles, different spatial dimensions, and other confinement potentials.
\end{abstract}

Keywords: confinement of quantum systems, $d$-dimensional calculations

PACS: 03.65.Ge, 21.45.-v

\section{Introduction}

The spatial dimension crucially determines the quantum mechanical properties of physical systems. In three dimensions a finite attraction is necessary to provide a bound state, whereas in two dimensions any infinitesimal attraction is enough to support a bound state $[1,2,3,4]$. This is most clearly seen for a system with one spatial coordinate, as for one particle in a potential or for the relative motion of two particles. The centrifugal barrier for such a simple system is zero or negative for $s$-waves in three and two dimensions, respectively.

It is conceptually easy to envisage confinement to lower dimensions than three, that is on planes or curves. Also deformed microscopic systems have been abundantly investigated, perhaps mostly in the many-body nuclear problem $[5,6,7,8]$, where symmetries can reduce the treatment to fewer than three spatial dimensions. Extreme deformations simulate lower dimensions like pancakes or cigar shapes. In nuclei these deformations are never extreme because they arise as self-

Email address: e.garrido@csic.es (E. Garrido) supported isolated structures without coupling to environment or other systems.

This is in sharp contrast to cold atoms and molecules where external fields are used to confine systems into deformed and even all the way to extreme lower dimensions $[9,10,11,12,13,14]$. The rapid technical developments in conjunction with the qualitative change of properties have prompted a number of both theoretical and experimental investigations in one and two dimensions [15, 16, 17]. However, in practical cold gas experiments the extreme limits of integer dimensions are only approximately achieved. The techniques apply to strong external fields which effectively can correspond to extreme deformations that only allow spatial extension in less than three dimensions.

Earlier attempts to study dimensional dependencies are available in particle and mathematical physics [18, 19, 20], and in the so-called $1 / d$-expansion [21, 22, 23]. In these methods the dimension $d$ is considered as an integer parameter, in such a way that for some particular values of $d$ the quantum mechanical solutions are available or readily obtained. Expanding the solutions around these particular $d$-values it is then possible to ex- 
trapolate the solution for the dimension of interest. The aim is to find correct converged solutions through this expansion. In this case all those solutions for $d$-values different from the one of interest have no physical meaning.

In this work the philosophy when introducing the dimension as a parameter is different. For practical reasons we focus here on dimensions between 2 and 3, where solutions can be obtained directly and accurately. Non-integer values of $d$ have now a physical interpretation, namely, they describe the confinement of the system, which can evolve continuously from moving in a three-dimensional to a two-dimensional space. This formulation has been available for a number of years for isolated systems, and especially detailed, but not exclusively, for two and three particles [3]. This formulation is precisely as efficient as ordinary integer dimension calculations, that is, as optimal as possible. However, the formalism is not connected to the available practical possibilities of laboratory tests, which on the other hand is conceptually straightforward by applying an external field to squeeze by gradually imposing confining walls.

The purpose of the present work is to provide the initial steps for a general and practically useful translation between the efficient non-integer $d$-formulation and the brute force method using an external field. For this we consider the relative motion of a two-body system, which has turned out to be interesting in itself, although first investigated as preparation for the much more complicated three-body system. Thus we first sketch the key points of the two methods, and second we provide interpretation and unique and universal translation between the methods. Finally we discuss the perspectives of applications to more complicated systems.

\section{Formulation}

The hamiltonian for two interacting particles in an external field in three spatial dimensions can be written as

$$
\begin{aligned}
H & =T+V(r)+V_{e x t}(z, Z), \\
T & =T_{r}+T_{R}, \\
V_{e x t}(z, Z) & =\frac{1}{2} \omega_{z}^{2}\left(M Z^{2}+\mu z^{2}\right),
\end{aligned}
$$

where we assume an external harmonic oscillator field in the $z$-direction, $V_{\text {ext }}(z, Z)$, of frequency $\omega_{z}$, acting on both particles and depending on the $Z$ and $z$ components of the center-of-mass and relative coordinates, $r$ and $R$, respectively, and on the total and reduced masses $M$ and $\mu$. We emphasize that the frequency is the same for both particles to ensure separation in relative and center-of-mass coordinates of both the external field and kinetic energy operator $T$. The particle-particle interaction, $V(r)$, will be assumed to be short-range.

The center-of-mass motion can now be solved completely independently, and in the following we shall concentrate on the relative coordinates. It is usual to give the oscillator frequency $\omega_{z}$ in terms of the oscillator length $b_{\text {ext }}=\sqrt{\hbar /\left(\mu \omega_{z}\right)}$, in such a way that the external potential can be written as

$$
V_{\text {ext }}(z)=\frac{\hbar^{2}}{2 \mu} \frac{z^{2}}{b_{e x t}^{4}},
$$

with $z=r \cos \theta$, where $\theta$ is the usual polar angle.

Note that the external potential (4) has a non-central character, which means that, as soon as $V_{\text {ext }} \neq 0$ the relative orbital angular momentum is not a good quantum number, and the total confined two-body wave function, $\Psi_{b_{e x t}}$, has to be obtained including a sufficient number of partial waves such that convergence is achieved, i.e.,

$$
\Psi_{b_{e x t}}(\boldsymbol{r})=\sum_{\ell m} \frac{u_{\ell}(r)}{r} Y_{\ell m}(\theta, \varphi) .
$$

In particular, the smaller the oscillator length $b_{\text {ext }}$, the larger the effect of $V_{e x t}$, and the higher the number of partial waves required. For $b_{\text {ext }}=\infty$, or $V_{\text {ext }}=0$, due to the central character of the two-body interaction $V(r)$, the quantum number $\ell$ is conserved and the expansion above reduces to one $\ell$-term.

The radial wave functions, $u_{\ell}(r)$, are obtained as the solution of the coupled set of differential equations:

$$
\begin{aligned}
& {\left[\frac{\partial^{2}}{\partial r^{2}}-\frac{\ell(\ell+1)}{r^{2}}-\frac{2 \mu}{\hbar^{2}}\left(V(r)-E_{t o t}\right)\right] u_{\ell}} \\
& -\frac{r^{2}}{b_{\text {ext }}^{4}} \sum_{\ell^{\prime} m^{\prime}}\left\langle Y_{\ell m}\left|\cos ^{2} \theta\right| Y_{\ell^{\prime} m^{\prime}}\right\rangle_{\Omega} u_{\ell^{\prime}}=0,
\end{aligned}
$$

where $E_{\text {tot }}$ is the total energy, and \langle\rangle$_{\Omega}$ indicates integration over the polar and azimuthal angles only. Note as well that $\left\langle Y_{\ell m}\left|\cos ^{2} \theta\right| Y_{\ell^{\prime} m^{\prime}}\right\rangle_{\Omega} \propto \delta_{m m^{\prime}}$, which implies that the orbital angular momentum projection $m$ along the $z$-axis remains as a good quantum number.

In this work we shall consider relative $s$-waves between the particles when $V_{e x t}=0$. This means that for any value of $b_{\text {ext }}$, the quantum number $m$ will be equal to zero. As a consequence, the dependence of the wave function (5) on the azimuthal angle $\varphi$ disappears.

This method is straightforward and simple, but generalization to three and perhaps more particles is quickly much more time consuming. To circumvent this complication we turn to another formulation [3, 24, 25] where the external field is omitted altogether, whereas 
the spherical two-body potential is maintained in $d$ dimensions. The relative Schrödinger equation is then

$$
\left[\frac{\partial^{2}}{\partial r^{2}}-\frac{(d-3)(d-1)}{4 r^{2}}-\frac{2 \mu}{\hbar^{2}}(V(r)-E)\right] R_{d}(r)=0
$$

where a relative $s$-wave has been assumed, $E$ is the energy, and $R_{d}(r)$ is the reduced radial wave function. The idea is now to vary $d$ continuously between $d=3$ and $d=2$, where the equation of motion in these two limits [3] are correct as seen by inspection of Eq. (7). This computation is much faster than by using the external field, especially for small values of $b_{\text {ext }}$, where Eq. (6) involves a large number of coupled differential equations.

The general form of Eq.(7) for integer values of $d$ contains an additional repulsive centrifugal term, i.e. $l_{d}\left(l_{d}+d-2\right) / r^{2}$, where $l_{d}$ is an integer, the angular momentum quantum number for $d=3$ and its projection for $d=2$. The corresponding angular wave functions are generalized spherical harmonics or related to Jacobi functions, where the coordinates are angles [3]. For $s$ waves such an angular dependence disappears. However, we can imagine excited and non-spherical states in both two and three dimensions. This extension to partial waves other than $s$-waves is definitely not trivial, since it necessarily at least implies an interpretation of $d-1$ angular variables, where $d-1$ may be a noninteger number. Such a generalization of the method is far beyond the scope of this report, but it is not entirely inconceivable for example by maintaining the azimuthal angle for $d \in[2,3]$, while gradually limiting the polar angle to a smaller interval than from 0 to $\pi$.

However, this research direction is not tempting, since the universal behavior of quantum systems is closely related to $s$-waves (or marginally also $p$-waves). Furthermore, large scattering lengths are necessary ingredients to have systems insensitive to the details of the potentials. Otherwise bound state wave functions would be pulled into the interior of the attractive potential and therefore loosing the universal character by being strongly dependent on the shape of the potential. For this reason, if a universal relation between the squeezing parameter $b_{\text {ext }}$ and the dimension $d$ exists, the restriction in Eq.(7) to relative $s$-waves appears very appropriate.

\section{Confinement length versus $d$}

The simplest characterizing quantities of any system are energy and radius, which can be used to give the connection between the parameters of the two methods, i.e., between $b_{\text {ext }}$ and $d$.
Table 1: Strengths, $S_{g}$ and $S_{m}$, for the three Gaussian (upper part of the table) and Morse (lower part of the table) potentials giving rise to the three-dimensional scattering lengths $a_{3 D}$. For each case we also give the $s$-wave two-body binding energies in $2 \mathrm{D}$ and $3 \mathrm{D}, E_{2 D}$ and $E_{3 D}$, and the corresponding root-mean square radii $r_{2 D}$ and $r_{3 D}$. All the energies are given in units of $\hbar^{2} / \mu b^{2}$ and the lengths in units of $b$, where $b$ is the range of either the Gaussian or the Morse potential.

\begin{tabular}{l|lll} 
& Pot. I & Pot.II & Pot.III \\
\hline$a_{3 D}$ & 2.033 & 18.122 & 40.608 \\
\hline$S_{g}$ & -2.71 & -1.43 & -1.38 \\
$E_{2 D}$ & -0.908 & -0.269 & -0.249 \\
$E_{3 D}$ & -0.269 & $-1.651 \cdot 10^{-3}$ & $-3.144 \cdot 10^{-4}$ \\
$r_{2 D}$ & 0.926 & 1.398 & 1.439 \\
$r_{3 D}$ & 1.508 & 12.823 & 28.710 \\
\hline$S_{m}$ & 1.294 & 0.474 & 0.434 \\
$E_{2 D}$ & -0.450 & $-7.394 \cdot 10^{-2}$ & $-6.088 \cdot 10^{-2}$ \\
$E_{3 D}$ & -0.189 & $-1.875 \cdot 10^{-3}$ & $-3.325 \cdot 10^{-4}$ \\
$r_{2 D}$ & 1.458 & 2.739 & 2.947 \\
$r_{3 D}$ & 2.235 & 12.870 & 28.741 \\
\hline
\end{tabular}

Two different shapes for the two-body potential $V(r)$ will be used: A Gaussian potential, $V(r)=S_{g} e^{-r^{2} / b^{2}}$, and a Morse-like potential, $V(r)=S_{m}\left(e^{-2 r / b}-2 e^{-r / b}\right)$. For each of them we have chosen three cases, each supporting one weakly bound two-body state, and whose three-dimensional (3D) identical scattering lengths, $a_{3 D}$, are given in Table 1. The strengths of the Gaussian and Morse potentials, $S_{g}$ and $S_{m}$, corresponding to each of the three $a_{3 D}$ values, are also given in the table, together with the $2 \mathrm{D}$ and $3 \mathrm{D}$ binding energies, $E_{2 D}$ and $E_{3 D}$, and root-mean-square radii, $r_{2 D}$ and $r_{3 D}$ for each case. We simplify by measuring lengths in units of the potential range, $b$, and energies in units of $\hbar^{2} / \mu b^{2}$.

Note that the value of $a_{3 D}$ increases significantly from potential I to III, implying a decrease of the binding energy $\left|E_{3 D}\right|$. The strength of the Gaussian and Morse potentials in the natural units in Table 1 approaches the limit $S_{g}=-1.34$ and $S_{m}=0.405$, respectively, corresponding to an infinitely large 3D scattering length. In this limit, the $2 \mathrm{D}$ scattering length, $a_{2 D}^{\infty}$, for the Gaussian and Morse potentials, takes the value 1.99 and 4.15, respectively, each in its corresponding length unit.

For the different potentials we then calculate the corresponding energies with the two methods as functions, respectively of the squeezing length $b_{e x t}^{b}=b_{\text {ext }} / b$ and the $d$-parameter. The corresponding results for the Gaussian (thick curves) and Morse (thin curves) potentials are shown in Fig. 1 normalized to the 2D energy. For the calculation using the external harmonic oscillator potential the energy $E$ is obtained after subtract- 

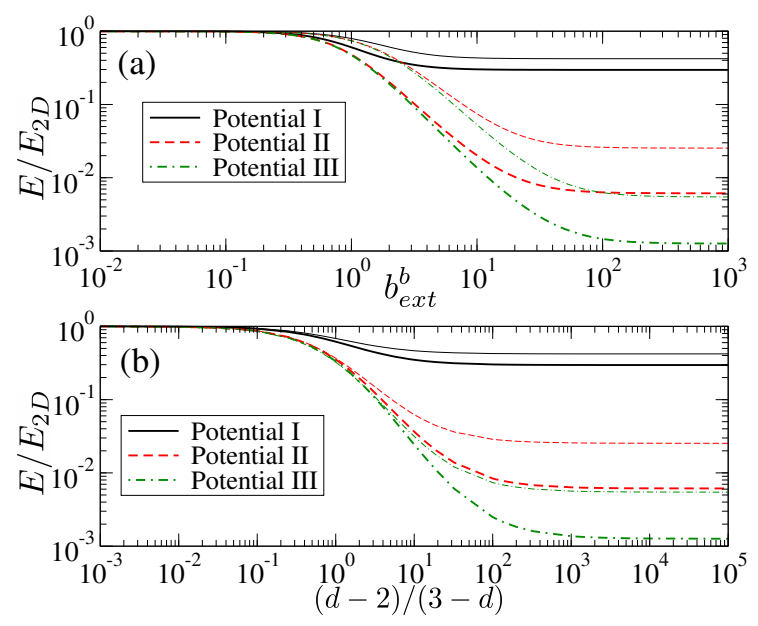

Figure 1: Two-body energies, normalized to the $2 D$-limit, for the Gaussian (thick curves) and Morse (thin curves) potentials in Table 1 The $x$-axis for panels (a) and (b) are the parameters used in the two methods, that is $b_{e x t}^{b}=b_{e x t} / b$ and $(d-2) /(3-d)$, respectively.

ing the zero point energy in the squeezed $z$-direction from the total computed energy in Eq.(6), i.e., $E=$ $E_{t o t}-\hbar \omega_{z} / 2$. By construction, all the curves reach unity in the $2 D$ limit, while the energies are much lower in $3 D$, where the values for both methods are $\hbar^{2} /\left(2 \mu a_{3 D}^{2}\right)$ for large scattering lengths.

The idea is now to relate $b_{\text {ext }}$ and the $d$-parameter by requiring that the energies are identical. For this to be useful we must find a model-independent translation. As we have learned from nuclear halo physics [3, 26, 27], and extensively as well in cold atomic gases [10, 28, 29], the appearance of a universal, modelindependent, behavior of quantum systems is associated to the presence of relative $s$-waves and large scattering lengths. This has been established as a universal parameter describing properties of weakly bound states without reference to the responsible short-range attraction. This is equivalent to the effective range expansion of the low-energy scattering phase shift, where the leading term is given by the scattering length. The next order entering with increasing energy depends on the range of the potential. The same properties are described by these quantities for disparate potentials. For this reason, the translation between $b_{\text {ext }}$ and $d$ obtained from Fig. 1 for the potentials with large scattering length should be very close to the desired universal relation between the two parameters. In fact, the precise relation should be provided by a potential with infinitely large scattering length.

The results are shown in Fig. 2a, where we show $b_{\text {ext }} / a_{2 D}^{\infty}$ as function of $d$ for the three potentials for the
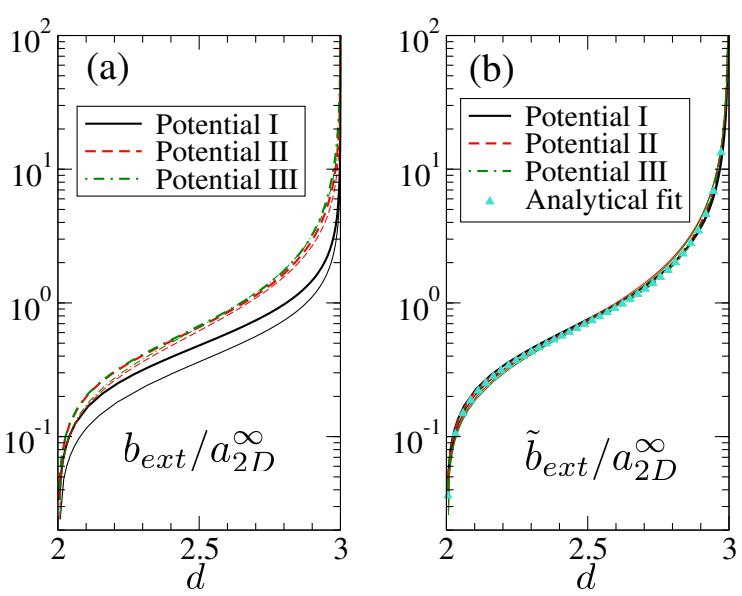

Figure 2: The derived dependence of $b_{\text {ext }} / a_{2 D}^{\infty}$ as function of $d$ in panel (a) for the potentials in Table 1 The thick and thin curves are the results for the Gaussian and Morse potentials, respectively. The universal curve in panel (b), parameterized in Eq.97, arises after use of the correction factor given in Eq. 8 .

Gaussian (thick curves) and Morse (thin curves) cases, where $a_{2 D}^{\infty}$ is the $2 \mathrm{D}$ scattering length obtained with a potential strength providing an infinite scattering length in $3 \mathrm{D}$. This amounts to using the same length unit for the two potential shapes, since, no matter the shape, the potential providing an infinite scattering length for $d=3$, gives rise to a scattering length, in the unit $a_{2 D}^{\infty}$, equal to 1 for $d=2$. As we can see, when the scattering length is large (potentials II and III), as expected, the short-range potential only has marginal influence, since the wave function is almost universal and outside the potential. The resulting translation is then already almost unique between $b_{\text {ext }} / a_{2 D}^{\infty}$ and $d$.

However, the uniqueness disappears when the scattering length in three dimensions is comparable to the potential range (potential I). This deficiency is to a large extent repaired by a correction factor, $\left(1+b_{\text {ext }} / a_{3 D}\right)$, which however, for small $a_{3 D}$ (potential I), still leaves small deviations for $d$ close to 2 and relatively small values of $b_{\text {ext }}$ comparable to the size of the system. The final fine-tuning is achieved after the transformation:

$$
\tilde{b}_{e x t}=b_{e x t}\left(1+\sqrt{\frac{b_{e x t}^{2}+r_{2 D}^{2}}{a_{3 D}^{2}}}\right),
$$

where $r_{2 D}$ (Table 1) is the root-mean-square radius for the bound state in two dimensions. This form is found by trial and error and not by an analytic derivation. However, the idea is as for effective range expansion in scattering theory, where the leading (universal) term is contained in the scattering length, while the next to 
leading order term is related to the range of the potential, which still is a universal average property.

The resulting efficient modification results in Fig. $2 \mathrm{~b}$ with the impressive reproduction for both potential shapes, as well as for small and large scattering lengths. The translation between $b_{\text {ext }}$ and $d$ only depends on gross properties as the scattering lengths in two and three dimensions and the mean-square-radius. These three quantities are all available by use of only calculations within the $d$-method. The translation is universal, or model independent, in the sense that it is independent of detailed properties of the potential. This is demonstrated by the results in Fig. 2 $b$ for rather different short-range potentials.

In Fig. 2 $\mathrm{b}$ we also show an analytical fit (triangles) that reproduces very well the universal curve. Since the curve is model independent, the special form of the fitting function is unimportant provided it is a sufficiently accurate fit which gives the connection between $\tilde{b}_{\text {ext }}$ and $d$. We have different options but one possibility is

$$
\frac{\tilde{b}_{e x t}}{a_{2 D}^{\infty}}=c_{1} \frac{d-2}{3-d}+c_{2} \tan \left((d-2)^{c_{3}} \frac{\pi}{2}\right),
$$

which is a combination of two functions, each of them being equal to zero at $d=2$, and to $\infty$ at $d=3$, and whose relative weight is used to fit the curves between these two limits. The computed fitting constants $c_{k}$ are $\left(c_{1}, c_{2}, c_{3}\right)=(-0.207,0.535,0.592)$.

It seems perhaps superfluous to use two terms with the same asymptotic behavior in both limits. However, each of them cannot reproduce intermediate dimensions. Analytic guidance is very difficult to find, since it involves details about how the excited modes in the direction perpendicular to squeezing are depopulated. Thus, not even the lowest correction term to the $d=2$ limit is simple. We leave this for possible future investigations.

It is important to note that after doing the calculations using the root-mean-square radii instead of the energy (Fig. 11), the same results as shown in Fig. 2, and therefore also in Eq. (9), are obtained. This is an indication of the robustness of the universal curve shown in Fig. 2 $\mathrm{b}$.

\section{Wave function interpretation}

The calculation of the two-body wave function in $d$ dimensions is formulated in spherical coordinates, where, since $s$-waves are assumed, the angles are absent. In particular, the corresponding wave function $\Psi_{d}$ takes the form:

$$
\Psi_{d}(r)=\frac{R_{d}(r)}{r^{(d-1) / 2}} Y_{d}, \text { with } Y_{d}=\left[\frac{\Gamma\left(\frac{d}{2}\right)}{2 \pi^{d / 2}}\right]^{1 / 2},
$$

where $R_{d}(r)$ is obtained from Eq.(7). The root-meansquare radius in $d$ dimensions is given by

$$
r_{d}^{2}=\int_{0}^{\infty} r^{2}\left|R_{d}(r)\right|^{2} d r
$$

This is a measure of the size of the wave function in $d$ dimensions, which unfortunately has no immediate physical interpretation. The mathematically well-defined concepts in $d$ dimensions are not directly applicable for computation of observables, which necessarily are measured in 3D. We therefore need an interpretation, or rather a translation, to be able to discuss ordinary three dimensional quantities.

In order to do so, we shall exploit the fact that we already have the comparison to the ordinary calculations with an external field, and the connection between $d$ and the external field parameter (Fig. 2). It is clear that the squeezing performed in one direction necessarily leads to a deformed structure. It looks then natural to try to reinterpret the total spherical wave function in $d$ dimensions, $\Psi_{d}$, as corresponding to a deformed threedimensional system.

To simulate the deformation produced by the external field, the most appealing procedure is to deform by scaling the radial coordinate, $r$, in the squeezed direction. We then take the usual Cartesian coordinates, $\{x, y, z\}$, in ordinary three dimensional space, and maintain the wave function, $\Psi_{d}$, obtained in the $d$-calculation, but where its radial argument is now substituted by

$$
r \rightarrow \tilde{r} \equiv \sqrt{x^{2}+y^{2}+(z / s)^{2}} \equiv \sqrt{r_{\perp}^{2}+(z / s)^{2}},
$$

where $s$ is a scaling parameter which depends on the geometry of the external potential, and in general might depend on $z$. The corresponding wave function, $\tilde{\Psi}_{d} \propto$ $\Psi_{d}$, must be normalized in the new three dimensional space, i.e.,

$$
2 \pi \int r_{\perp} d r_{\perp} d z\left|\tilde{\Psi}_{d}\left(r_{\perp}, z, s\right)\right|^{2}=1 .
$$

It is clear that the limits of $d=2$ and $d=3$ are correct. For instance, for $s=0$ only $z=0$ is possible (otherwise $\tilde{r}=\infty$ and $\left.\Psi_{d}(\infty)=0\right)$ and we are in the $d=2$ situation. Similarly, for $s=1$ the relative coordinate $\tilde{r}$ is the usual radius in spherical coordinates, and we are in the $d=3$ case. 

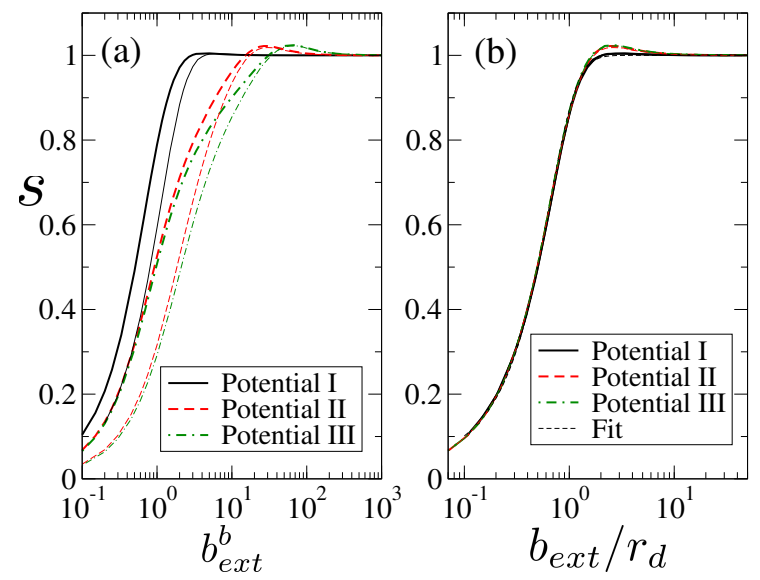

Figure 3: The scaling parameter $s$ as a function of $b_{\text {ext }}^{b}=b_{\text {ext }} / b$ (panel a) and as a function of $b_{\text {ext }} / r_{d}$ (panel $\mathrm{b}$ ) for the potentials in Table 1 The thick and thin curves correspond to the Gaussian and Morse potentials, respectively. In panel (b) the curve denoted as "Fit" (thindashed) corresponds to the analytical fit in Eq. 15.

The suitability of this procedure can be tested analytically by the rather efficient one-gaussian approximation [30]. We compare the best one-gaussian wave functions for the same energy from the external field, $R_{\text {ext }} \propto \exp \left(-r_{\perp}^{2} /\left(2 b^{2}\right)-z^{2} /\left(2 b_{z}^{2}\right)\right)$, and the $d$ calculations, $R_{d}(r) \propto \exp \left(-r^{2} /\left(2 b_{d}^{2}\right)\right)$, where $R_{\text {ext }}$ is the wave function without phase factor, and $r$ is scaled as in Eq. (12). The maximum overlap of unity is obtained for $b=b_{d}$ and $s=b_{z} / b_{d}$. This observation is very encouraging, although not surprising due to the analogous one-gaussian assumptions, which often is rather efficient and therefore making it even more appealing to exploit.

\section{Wave function parameterization}

We generalize by comparing the wave functions from the $d$-calculation and the full external field, $\Psi_{b_{e x t}}(\boldsymbol{r})$ in Eq. (5), where $r^{2}=r_{\perp}^{2}+z^{2}$. The overlap between these functions is defined by

$$
O(d, s)=2 \pi \int r_{\perp} d r_{\perp} d z \tilde{\Psi}_{d}\left(r_{\perp}, z, s\right) \Psi_{b_{e x t}}(\boldsymbol{r})
$$

where $s$ is adjusted to produce maximum overlap for the given $d$, which in turn is universally related to $b_{\text {ext }}$ through Eq. 9), see Fig. 2b for accuracy.

The computed values of the scale parameter $s$ for the potentials used in this work are shown in Fig. 3a as a function of $b_{\text {ext }}^{b}$. The larger the squeezing the smaller the scale parameter which, as anticipated, varies between 0 and 1. Only in the region of little squeezing $s$ takes values slightly larger than 1 . This fact indicates that in this region ( $d$ very close to 3 ) the interpretation of the $d$-wave function as $\tilde{\Psi}_{d}\left(r_{\perp}, z, s=1\right)$ gives rise to a state with the particles a bit too confined along the $z$ axis, in such a way that maximization of the overlap (14) requires a small release of the confinement by means of a scale factor bigger than 1 .

The differences between the curves shown in Fig. 3 a are related to the size of the two-body system. For both, the Gaussian and Morse shapes, potential III is the one with the largest scattering length in $3 \mathrm{D}$, and therefore the one describing the largest two-body bound state. For this reason, the curve corresponding to this potential is the first one feeling the squeezing, i.e., it is the first one for which $s$ deviates from 1 when the squeezing parameter $b_{\text {ext }}^{b}$ is made smaller and smaller. For the same reason the second potential feeling the squeezing is potential II, and for potential I the deviation from $s=1$ starts for even smaller values of $b_{\text {ext }}^{b}$. A simple way to account for these size effects is to plot the scale parameter $s$ as a function of $b_{\text {ext }} / r_{d}$, where $r_{d}$ is the rms radius of the system for dimension $d$ as given in Eq.11).

The result is shown in Fig. 3b, and, as we can see, all the curves collapse into a single universal curve, which therefore permits to relate the scale parameter $s$ and the squeezing parameter $b_{\text {ext }}$ (or $d$ through Eq.(9)) independently of the potential. Only some small discrepancy is observed for potential I in the region of little squeezing, where $s>1$. The emerging universal curve is very satisfying. We emphasize the crucial point that the universal curve is defined and obtained entirely within the practical $d$-calculation.

A simple analytic fit to the universal curve in Fig. $3 \mathrm{~b}$ is given by

$$
\frac{1}{s(x)}=1+c_{1} \frac{e^{-c_{2} x}}{x^{c_{3}}} ; \quad x=\frac{b_{e x t}}{r_{d}},
$$

with $c_{1}=2.75, c_{2}=2.92$, and $c_{3}=0.64$, which gives rise to the fitted and hardly distinguishable thin-dashed curve in Fig. 3 $\mathrm{b}$. The transition between $d=2$ and $d=3$ is exponentially fast in the region where the squeezing length is comparable to the size of the system. The result in Eq.15) is again, as Eq.(9), an accurate but somewhat arbitrary fitting function with precisely the same difficulties in deriving analytically.

It is important to recall the parameter $s$ has been obtained for each $d$ such that the overlap in Eq. (14) is maximum. Obviously, for $d=3$ the two wave functions involved in Eq. (14) are solutions of the same equation, and therefore the overlap is maximum and exactly 1 for $s=1$. However, under a large squeezing situation the equations giving rise to $\tilde{\Psi}_{d}$ and $\Psi_{b_{e x t}}$ are superficially 

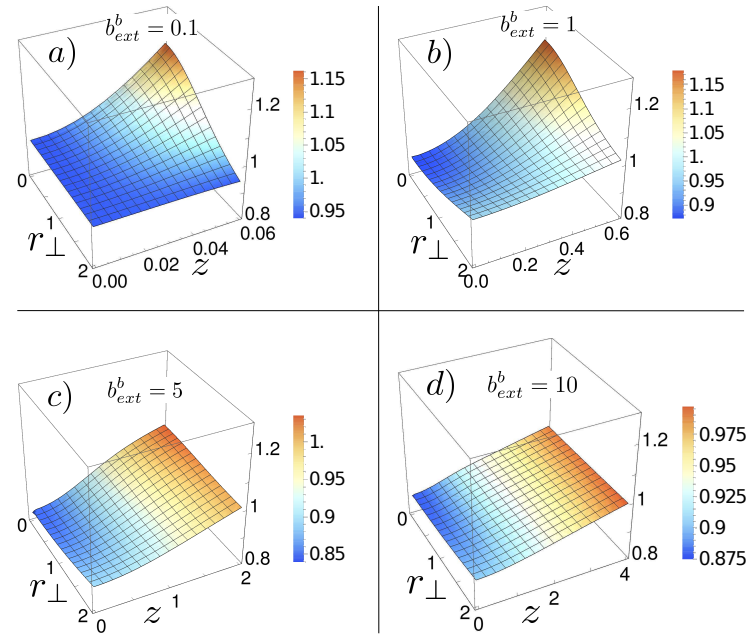

Figure 4: For the Gaussian potential II, ratio between the wave functions $\Psi_{b_{e x t}^{b}}(r)$ and $\tilde{\Psi}_{d}\left(r_{\perp}, z, s\right)$ for four different values of $b_{e x t}^{b}$, as a function of $r_{\perp}$ and $z$ in units of $b$. The maximum value shown for the $z$-coordinate is given by $\left\langle z^{2}\right\rangle^{1 / 2}$.

very different, and therefore the overlap, even if maximized, might differ from 1 .

Also, for $d=2$, both wave functions describe a spherical two-dimensional structure obtained with the same interaction, and can correspondingly be expected identical. This is confirmed when $s$ is chosen to maximize the overlap, which even in the most unfavorable computed case $\left(b_{\text {ext }}^{b}=0.1\right.$, i.e., $d$ and $s$ approaching 2 and 0 , respectively) is numerically found equal to $0.993,0.984$, and 0.984 , for the Gaussian potentials I, II, and III, and 0.989, 0.980, and 0.977 for the Morse potentials I, II, and III, respectively. This result supports the interpretation of $\Psi_{d}$ described above for the intermediate interval of $d$ between 2 and 3 .

Instead of the very stable overlap value, we show in Fig. 4 for the Gaussian potential II, the more sensitive ratio $\Psi_{b_{e x t}} / \tilde{\Psi}_{d}$ as a function of $r_{\perp}$ and $z$ (in units of $b_{b}$ ). Panels $a, b, c$, and $d$ show, respectively, the ratio obtained for squeezing parameters covering the whole range of dimensions under investigation, that is $b_{\text {ext }}^{b}=0.1, b_{\text {ext }}^{b}=1.0, b_{\text {ext }}^{b}=5.0$, and $b_{\text {ext }}^{b}=10.0$. For each of the cases, the plots extend to the largest $z$-value taken to be $\left\langle z^{2}\right\rangle^{1 / 2}$, where the wave functions only below this value differ significantly from zero. The scale in the four panels has been chosen to emphasize the differences between them. In any case, the ratio in Fig. 4 is rather stable and close to 1, until the far corner where each wave function is vanishingly small. For this reason, these deviations are of no consequence, since they are obviously inaccurate as obtained in the limit of zero divided by zero. The same level of similarity (not shown) is found for the other potentials. This is a conclusive proof of our interpretations.

\section{Procedure in $d$ dimensions}

The interpretation of $\Psi_{d}$ allows full calculation of any desired observable, entirely within the efficient $d$ calculation. In other words the otherwise undefined angular part is now well-defined through the translation and the universal curves. For instance, we can compute the partial wave content in the usual way by means of the expansion

$$
\tilde{\Psi}_{d}\left(r_{\perp}, z, s\right)=\sum_{\ell, m} R_{\ell}(r) \mathcal{Y}_{\ell m}(\theta, \phi)
$$

where the radial function reveals the weight of each partial wave.

As discussed in section 2, in our case of a squeezing potential with axial symmetry, $m$ is a good quantum number, and since only $s$-waves are considered, we then have $m=0$, and the partial wave components in the wave function (16) will depend only on $r$ and the polar angle $\theta=\arctan \left(r_{\perp} / z\right)$. It is therefore evident how any observable can be computed via only $d$-calculated quantities.

Summarizing, given a two-body system interacting via a potential $V(r)$ with scattering length $a_{3 D}$, and put under the effect of an external potential with oscillator length $b_{\text {ext }}$, the procedure is as follows: i) Calculate the key quantities, i.e. rms radius of the bound state in two dimensions, and the 2D scattering length when $a_{3 D}=\infty$. ii) get the corresponding dimension $d$ from the analytic expression (9), or Fig. 2b, iii) obtain $\Psi_{d}$ and $r_{d}$ in Eqs.10 and (11) after solving the Schrödinger Eq. (7) for that particular dimension, iv) find the scale parameter, $s$, from the analytic expression in Eq. (15), or Fig. 3 , by use of the ratio of $b_{e x t} / r_{d}$, and finally, v) use the wave function (16) in order to compute whatever observable is of interest.

It is perhaps fair to issue the warning that choosing another external squeezing field may change the results. We anticipate that the scaling parameter is constant, independent of $z$, because the external oscillator potential is quadratic in $z$, that is our scaling of the squeezed coordinate. For another potential a new investigation must be carried out, but such generalization to other shapes is far beyond the scope of the present letter. 


\section{Perspectives and Conclusions}

Squeezing by an external field and a dimension $(d)$ dependent centrifugal barrier are two formulations to account for a continuous variation of the spatial dimension between ordinary integer values. We have demonstrated that the methods are equivalent for two-body systems with a unique and universal correspondence between external field and the $d$-parameter. The relations are relatively simple and can be expressed approximately by an analytic formula. The $d$-formulation is much simpler, both smaller in computer space and time, and therefore more efficient. In fact, the method is precisely as complicated as an ordinary three dimensional calculation with a centrifugal barrier, that is using only relative coordinates. Thus, the wave function with an external field in an ordinary 3D calculation is easily obtained.

Clearly this is not of great consequence for simple two-body systems. However, the brute force method with squeezing by an external field is already much more difficult for a three-body system than the already mathematically available $d$-method. The interpretation and equivalence to an external field only has to be worked out and tested. However, this is not an easy matter and it requires an investigation on its own. Especially mass asymmetric systems with several and more complicated excited states is a challenge and must be handled.

We treated the most important transition from three to two spatial dimensions, but different continuous variation of dimension can be suggested, like from two to one and further on down to zero dimension. Beside doing the squeezing on one dimension at a time it is possible to go directly from three to one (or zero) by for example squeezing two (or all three) directions simultaneously. Then the integer dimension of two (or one) would never be passed, but the $d$-formulation is still valid although the interpretation and the equivalence translation must change. The actual calculations are in all these cases still equally simple and dealing with only relative coordinates.

The key to the technical simplifications is the translation from the external field to the $d$-formulation. We provide a simple and direct guide to go from $d$ calculations to ordinary three dimensional quantities, still only using $d$-quantities like energies and wave functions. We have chosen to investigate in details the use of an oscillator field as it appears to be both relatively simple and also practically achievable in laboratories. However, other external fields can also be studied as for example a square well squeezing where the interior of the squeezed directions have zero potential and only the walls cause the dimensional confinement. As the experimental techniques rapidly develop other shapes may come in as interesting.

In summary, we have for a simple system demonstrated how the efficient calculations with the $d$ formulation are equivalent to the more complicated results obtained by squeezing with an external field. The energies and wave functions obtained with the $d$-method are for each $d$ demonstrated to be uniquely related to the parameter, $b_{\text {ext }}$, of the external field. We illustrated by using both Gaussian and Morse potentials. Thus, the $d$-method provides the same full information as a brute force three dimensional calculation with the external field.

\section{Acknowledgements}

This work has been partially supported by the Spanish Ministerio de Economía y Competitividad under Projects FIS2014-51971-P, FPA2015-65035-P and MTM2015-63914-P.

\section{References}

\section{References}

[1] B. Simon, Annals of Phys. 97 (1976) 279

[2] L.D. Landau and E.M. Lifshitz, Quantum mechanics: Nonrelativistic theory, Pergamon Press Ltd. (1977) p.163

[3] E. Nielsen, D.V. Fedorov, A.S. Jensen and E. Garrido, Phys. Rep. 347 (2001) 373

[4] D. Petrov and G. Shlyapnikov, Phys. Rev. A 64 (2001) 012706

[5] N.T. Zinner, Few-body Syst. 55 (2014) 599

[6] O. Sørensen, D.V. Fedorov and A.S. Jensen, Phys. Rev. Lett. 89 (2002) 173002

[7] M. Thøgersen, D.V. Fedorov and A.S. Jensen, Europhys. Lett. 83 (2008) 30012

[8] D. Hove, E. Garrido, P. Sarriguren, D.V. Fedorov, H.O.U. Fynbo, A.S. Jensen and N.T. Zinner, J. Phys. G: Nucl. Part. Phys. 45 (2018) 073001

[9] C.E. Klauss, X. Xie, C. López-Abadía, J.P. DIncao, Z. Hadzibabic, D.S. Jin and E.A. Cornell, Phys. Rev. Lett. 119 (2017) 143401

[10] P. Naidon and S. Endo, Rep. Prog. Phys. 80 (2017) 056001

[11] J. Levinsen, P. Massignan and M.M. Parish, Phys. Rev. X 4 (2014) 031020

[12] J.H. Sandoval, F.F. Bellotti, A.S. Jensen and M.T. Yamashita, Phys. Rev. A 94 (2016) 022514

[13] J.H. Sandoval, F.F. Bellotti, A.S. Jensen and M.T. Yamashita, J. Phys. B: At. Mol. Opt. Phys. 51 (2018) 065004

[14] E.R. Christensen, A.S. Jensen and E. Garrido, Few-body Syst. 59136

[15] E. Nielsen, D.V. Fedorov and A.S. Jensen, Phys. Rev. A 56 (1997) 3287

[16] J.K. Pedersen, D.V. Fedorov, A.S. Jensen and N.T. Zinner, J. Phys. B: At. Mol. Opt. Phys. 49 (2016) 024002 
[17] R.E. Barfknecht, A. Foerster and N.T. Zinner, Few-body Syst. 59 (2018) 22

[18] G. Leibrandt, Rev. Mod. Phys. 47 (1975) 849

[19] A. Gonzalez, Few-body Systems 13 (1992) 105

[20] M. Dunn, D.K. Watson, Phys. Rev. A 59 (1999) 1109

[21] D.J. Doren and D.R. Herschbach, Phys. Rev. A 34 (1986) 2654

[22] D.R. Herschbach, J. Avery and O. Goscinski (eds.) Dimensional Scaling in Chemical Physics, Kluwer Academic Publishers, Dordrecht (1992)

[23] M. Dunn, D.K. Watson, in D.R. Herschbach, J. Avery and O. Goscinski (eds.) Dimensional Scaling in Chemical Physics, Kluwer Academic Publishers, Dordrecht (1992)

[24] D.S. Rosa, T. Frederico, G. Krein and M.T. Yamashita, Phys. Rev. A 97 (2018) 050701

[25] D.S. Rosa, T. Frederico, G. Krein, and M.T. Yamashita, J. Phys. B: At. Mol. Opt. Phys. 52 (2019) 025101

[26] A.S. Jensen, K. Riisager, D.V. Fedorov, and E. Garrido, Rev. Mod. Phys. 76 (2004) 215

[27] T. Frederico, A. Delfino, L. Tomio and M.T. Yamashita Prog. Part. Nucl. Phys. 67939

[28] E. Braaten and H.-W. Hammer Phys. Rep. 428 (2006) 259

[29] T. Frederico, L. Tomio, A. Delfino, M.R. Hadizadeh and M.T. Yamashita Few-body Syst. 51 (2011) 87

[30] The one-gaussian approximation is the best variational wave function with only one gaussian minimizing the expectation value of a given hamiltonian. 